\title{
Assoziation zwischen Hautjucken und Krebs
}

\author{
Eignet sich Juckreiz als Marker für eine Krebserkrankung? \\ Obwohl viele Krebspatienten, besonders solche mit malignen \\ hämatologischen Erkrankungen, unter Juckreiz leiden, liegen \\ nur begrenzte Daten über die Eignung als Marker für eine \\ Krebserkrankung vor. \\ $\mathrm{Br}$ ] Dermatol 2014; 171: 839-846
}

Die dänischen Wissenschaftler um S.A. Johannesdottir haben die Inzidenz von Krebs bei Patienten mit Pruritus untersucht. Unter Verwendung von medizinischen Datenbanken wie dem Danish National Registry of Patients (DNRP) und dem Danish Cancer Registry (DCR) identifizierten die Autoren Patienten, bei denen zwischen 1978 und 2011 Pruritus diagnostiziert wurde, und verfolgten diese bis zu einer ersten Krebsdiagnose bzw. bis zur Emigration, zum Tod oder bis Ende 2011. Dabei handelte es sich um hospitalisierte, ambulante und Notfallpatienten. Es wurden jeweils die standardisierten Inzidenzverhältnisse (Standard Incidence Ratio, SIR) sowie das absolute 1-Jahres-Krebsrisiko ermittelt.

\section{Erhöhte Krebsinzidenz nach Pruritus-Diagnose $\nabla$}

In die Analyse gingen die Daten von 97968 Personen ein. Davon wurden 12813 Patienten mit Pruritus identifiziert. Das Verhältnis von Männern zu Frauen betrug $3: 4$, das mediane Alter der Studienteilnehmer lag bei 53 Jahren (Interquartile Range $[\mathrm{IQR}]=36-70)$. Während des Follow-ups wurden 1173 Krebsfälle identifiziert. Nach Berechnungen der nationalen Kerbsinzidenz in Dänemark bezogen auf die Personenjahre des Studienzeitraums wären 1039 Fällen zu erwarten gewesen. Dies ergab eine SIR von 1,13 (1,22 unter den Männern und 1,05 unter den Frauen). Im Fall von Pa- tienten mit einer im Vorfeld diagnostizierten Hauterkrankung lag die SIR bei 1,20 gegenüber 1,10 bei Patienten ohne eine solche Diagnose. Sowohl hämatologische Krebserkrankungen als auch verschiedene Arten von soliden Tumoren traten in verstärktem Maße auf. Die höchste SIR war während der ersten 3 Monate der Nachbeobachtung zu verzeichnen $(2,14)$, der Wert sank während des Zeitraums von 4-12 Monaten rasch auf 1,42. Das absolute 1-Jahres-Krebsrisiko lag bei $1,63 \%$. Es hätten also 155 Pruritus-Patienten untersucht werden müssen, um einen zusätzlichen Krebsfall zu identifizieren.

Fazit
Pruritus-Patienten wiesen innerhalb
von 3 Monaten nach der Diagnose eine
2-fach erhöhte Krebsinzidenz auf. Diese
sank anschließend allerdings rapide.
Das absolute 1-Jahres-Krebsrisiko belief
sich auf 1,63\%. Um einen zusätzlichen
Krebspatienten zu identifizieren, wäre
es nötig gewesen, 155 Patienten mit
Juckreiz zu untersuchen. Auf Basis der
aktuellen Datenlage empfehlen die Au-
toren bei Patienten mit Pruritus keine
umfangreiche diagnostische Abklärung
hinsichtlich einer möglichen Krebser-
krankung.

\section{Dr. Frank Lichert, Weilburg}

rer jüngsten Publikation Ende 2014 in der Fachzeitschrift BioMetal konnten die Forscher erstmals für solche Peptid-Derivate eine hohe spezifische Aktivität für eine Melanom-Zelllinie und Melanom-Metastasen aufzeigen sowie einen eindeutigen Beweis für die Interaktion der Peptide mit dem Krebsmarker PS liefern. „Die Lactoferricin-Derivate weisen gegenüber dem Ausgangspeptid eine über 10-fach erhöhte Toxizität für Hautkrebszellen auf, während sie gesunden Zellen genauso wenig schaden wie Lactoferricin“, so Zweytick. Darüber hinaus wurde bewiesen, dass die Struktur der modifizierten Proteinmoleküle eine wesentliche Rolle spielt: „Peptide mit einer Helix-Struktur wirken sehr stark, aber unspezifisch“, erklärt Zweytick. Peptide mit einer Haarnadel-Struktur bräuchten zwar länger, seien dafür aber hoch selektiv und lösten den natürlichen Zelltod aus.

Nach einer Mitteilung der Karl-FranzensUniversität Graz 